\title{
Three-dimensional modeled environments versus 360 degree panoramas for mobile virtual reality training
}

\author{
K. A. Ritter III' ${ }^{1}$. Terrence L. Chambers ${ }^{1}$
}

Received: 10 September 2019 / Accepted: 18 January 2021 / Published online: 10 March 2021

(c) This is a U.S. government work and not under copyright protection in the U.S.; foreign copyright protection may apply 2021

\begin{abstract}
Virtual field trip is a way of providing users with some knowledge and exposure of a facility without requiring them to physically visit the location. Due to the high computational costs that are necessary to produce virtual environments (VEs), the potential for photorealism is sacrificed. Often these three-dimensional (3D) modeled applications use an unrealistic VE and, therefore, do not provide a full depiction of real-world environments. Panoramas can be used to showcase complex scenarios that are difficult to model and are computationally expensive to view in virtual reality (VR). Utilizing $360^{\circ}$ panoramas can provide a low-cost and quick-to-capture alternative with photorealistic representations of the actual environment. The advantages of photorealism over 3D models for training and education are not clearly defined. This paper initially summarizes the development of a VR training application and initial pilot study. Quantitative and qualitative study then was conducted to compare the effectiveness of a $360^{\circ}$ panorama VR training application and a 3D modeled one. Switching to a mobile VR headset saves money, increases mobility, decreases set-up and breakdown time, and has less spatial requirements. Testing results of the 3D modeled VE group had an average normalized gain of 0.03 and the $360^{\circ}$ panorama group, 0.43 . Although the 3D modeled group had slightly higher realism according to the presence questionnaire and had slightly higher averages in the comparative analysis questionnaire, the $360^{\circ}$ panorama application has shown to be the most effective for training and the quickest to develop.
\end{abstract}

Keywords Virtual training · Virtual reality · Virtual laboratories $\cdot$ Virtual field trip

\section{Introduction}

Virtual reality (VR) training provides a portable solution for learning and refinement of skills that reduces costs associated with bringing in specialized educators and travel time as well as risk to the student. Integrating workplace competencies into an application with strategies to support student retention can potentially lead to improved technical science, technology, and engineering (STE) education. Twenty-one experimental studies involving VR training since 2013 were analyzed and found that head-mounted devices (HMD) $\mathrm{s}$ are useful for skills acquisition, such as cognitive skills related to remembering and understanding spatial and visual information and knowledge; psychomotor skills related to head movement, such as visual scanning or observational

\section{K. A. Ritter III}

kritter@louisiana.edu

1 Department of Mechanical Engineering, University of Louisiana at Lafayette, Lafayette, LA, USA skills; and affective skills related to controlling emotional responses to stressful or difficult situations (Jensen and Konradsen 2017). As the decay of a skill depends greatly on the degree to which the skill was learned, the higher the acquisition environment (e.g., immersive training), the longer the retention (Loftus 1985). This approach can increase the skilled workforce while decreasing training costs and safety concerns. As the technologies in industry are rapidly changing, providing suitable training programs is of utmost importance. Computer-based learning and other traditional training programs are not adequate in training for various situations with which decision makers must deal (Wang et al. 2018). On-the-job training is not practical with high cost and safety concerns in many on-site work situations that have a high focus on productivity. A meta-analysis of nearly 70 educational VR applications gives evidence that VR-based training is an effective means of enhancing learning outcomes (Merchant et al. 2014). Virtual reality training facilitates experiential learning, which is referred to as learning through action, learning by doing, learning through 
experience, and learning through discovery and exploration (Kolb and Kolb 2005). In VR, users are immersed in a reallife simulation and learn through practical experience. Studies have shown that learning through experience increases the quality of learning and retention by $75-90 \%$ and has been argued as being the most effective way to learn (PérezSabater 2011). Virtual reality training applications can give future engineers the convenience of experiencing complex processes visually by viewing animations in an immersive environment (Ritter III et al. 2018). There has been a consistent increase in publications in VR training in the last 20 years as shown in Fig. 1.

Publishing articles on VR that involve training has climbed from six in 1998 to 213 in 2018 and will reach over 300 in 2020 following this trend. Currently, VR training systems are used in many different fields, such as manufacturing, medicine, education, and military, as well as training programs for skills of professionals, employees, specialists, and managers in prototyping, assembly, drive, flight, and surgery, among others (Borsci et al. 2015). Virtual reality has been shown to improve education in a number of ways. A shader script was developed that automatically renders complex object meshes to fit entirely within the field of view of consumer head-mounted displays and has shown an increase in speed that users take to complete tasks (Cook and Grime 2020). An interactive VR heart anatomy system showed a higher satisfaction rate of the structure and visualization tools when compared to traditional modalities of learning anatomy (Alfalah et al. 2018). A higher sense of spatial presence and immersion has been shown while using head-mounted displays when compared to desktop (Shu et al. 2019). In an immersive learning study involving large stellar structure interaction, a significant advancement is shown on one's perception of Newton mechanics on largescale stellar bodies (Datallo et al. 2018). In product design and manufacturing, advantages of VR have been shown with visibility, ergonomics, packaging, aesthetic quality, abstract

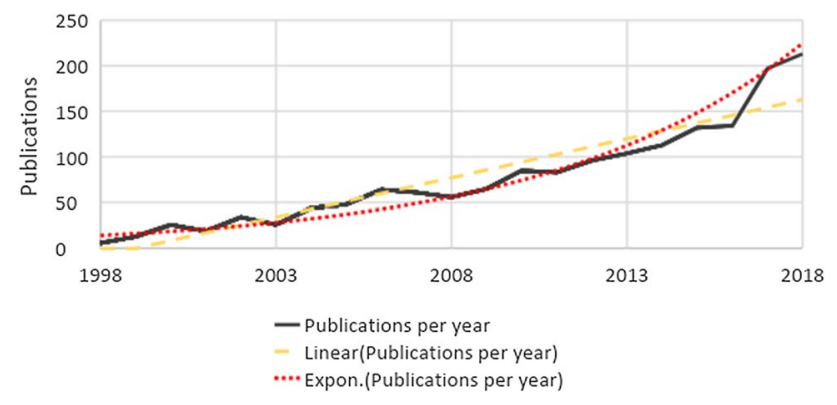

Fig. 1 Number of articles per year with titles that contains virtual reality and on the topic of "Training" in Web of Knowledge from 1998 to 2018. The linear trend shows the constant growth, while the exponential trend estimated the expected growth data visualization, storytelling, as well as across discipline communication (Berg and Vance 2017).

Using VR headsets in virtual environments (VEs) allows users to be immersed and interact with objects in the VE. Interactions can play a crucial role in learner engagement, and developing interactive learning activities is of high priority for effective VE education (Christopoulos et al. 2018). VE's modeled in three dimensions (3D) have limited capabilities of delivering high degrees of realism, which might limit the effectiveness of virtual training applications. Modeling photorealistic environments in 3D takes a significant amount of effort in terms of time and has a high computational cost for rendering all the elements in the scene (Eiris et al. 2018). In contrast, $360^{\circ}$ panorama-based VEs offer low-cost, easy-to-capture, non-computer-generated simulations that can provide a true-to-reality representation of the environments (Eiris et al. 2020). These have the potential to overcome many of the limitations of 3D modeled-based VEs. These full panoramas can be used to create a VE that can provide unbroken views of a whole region surrounding an observer. The application of $360^{\circ}$ panoramic videos offers a unique sense of presence and immersion that has potential in teaching and learning (Reyna 2018). Interactive panoramic scenes also have been used for several applications by researchers for education and training. Virtual safety training in the construction industry has utilized 3D modeled VEs (Le et al. 2015; Li et al. 2012; Sacks et al. 2013) and $360^{\circ}$ panorama technology (Eiris et al. 2018; Pereira et al. 2018; Pham et al. 2018). For hazard identification in construction sites, a 3D modeled and $360^{\circ}$ panorama were compared in a pilot study and users identified slightly more hazards in the 3D modeled condition (Moore et al. 2019). Furthermore, in a qualitative assessment, the users in the Moore study found 3D modeled to be easier and clearer than the $360^{\circ}$ panorama. This could be due to the image quality of commercially available $360^{\circ}$ cameras which are not yet comparable to traditional videography. The quality of multiple cameras is degraded due to issues with misalignment and discontinuity caused from the parallax between cameras (Lee et al. 2016). In perceptions of safety and security, $360^{\circ}$ videos were found to elicit the same assessments as actually being there (Rossetti and Hurtubia 2020). The best psychological outcome scores were found with the $360^{\circ}$ panoramas when compared to a 3D modeled environment, and a static image (Higuera-Trujillo et al. 2017).

Leaving the classroom and engaging in learning through firsthand experience has a long tradition in science and engineering education. However, it is difficult to organize adequate field trips for all students in various courses due to time, distance, and safety issues. Virtual laboratories can provide remote access to various disciplines of STE and are a cost-efficient way for schools and universities to organize high-quality laboratory work. 
Due to restrictions on time and geographical distances, virtual laboratories can be used to share costly equipment and resources, which otherwise are unavailable to a majority of users. The use of VR training applications, such as virtual laboratories, is a growing research area for professionals and operators in many different fields. Laboratory exercises are required in many fields to provide effective skill acquisition and hands-on experience. As access to these laboratories for training is not always feasible for many trainees, replicating the laboratory as a fully software-based virtual laboratory could potentially solve this issue. In a review of virtual laboratories, several advantages were identified, including cost savings, flexibility in experiments, multiple user access, and damage resistance. Also, visual advantages, such as transparent equipment covers that reveal motors, gearboxes, and other components, were noted (Potkonjak et al. 2016). One study compared actual field trips with immersive virtual field trips (iVFTs) and identified many advantages with the iVFTs, including reductions in cost and time; avoiding weather issues, physical inaccessibility, or dangerous places; providing access to online teaching environments; and scale, as many field trips are spatially constrained (Klippel et al. 2018). Therefore, many practical problems in education and training can be addressed via VR training applications.

The Photovoltaic (PV) Applied Research and Testing (PART) Lab encompasses a 1.1-MW PV power plant with three solar panel technologies, meteorological and radiometer stations, and PV testing laboratory. Using a scale model of the PV power plant, PV-VR is an application that provides educational interactive tours in VR. Higher student engagement and knowledge has been shown for solar energy education using a cloud-based VR system (Abichandani et al. 2019). The actual PV solar power plant is virtualized, and virtual representations of all the objects in the plant are inserted into the application. This PV-VR takes users on a guided educational tour that allows them to interact with the various technologies to facilitate virtual hands-on learning. The virtual tour is guided by a teacher avatar that explains each technology and initiates animations and interactive educational games with constructive feedback to reinforce learning. The PV-VR allows users to learn about solar power technologies, and how power is produced, converted, and transmitted.

The initial aim of this research is to develop and test a 3D modeled iVFT and perform a pilot study to refine and improve it. The secondary aim is to develop the same VE using $360^{\circ}$ panoramas and gauge the effectiveness with a quantitative and qualitative comparative analysis study. The two separate studies have been performed on two different participant groups and results presented.

\section{Method}

\subsection{Developing the PV-VR iVFT}

The PV-VR is a guided interactive virtual tour of an existing solar power plant. The application consists of several interactive educational areas intended to give students a brief overview of the solar resource, PV chemistry, direct current (DC)-to-alternating current (AC) inverters, various PV technologies, measurement devices, and the power and distribution network at the PART Lab. The actual PART Lab, shown in Fig. 2, is situated near the University of Louisiana at Lafayette and tests several solar technologies side by side. The PART Lab is a power-producing plant that tests multiple solar technologies, including polycrystalline, monocrystalline, and thin film, simultaneously.

The PV-VR application is designed to give virtual tours of the PART Lab while explaining the various solar technologies. This application was developed using the game engine, Unity (Unity 3D 2019); the digital audio editor, Audacity (Audacity 2019); and the 3D computer graphics applications of Solidworks (Dassault Systèmes SolidWorks Corporation 2018), Blender (2019), and Maya (Autodesk 2019). Unity is the game engine that provided the platform for adding models, scripts, animations, and building the application. The Unity plugin VRTK (VR Tool Kit 2019) provided grabbing interactions and controls. Blender and Maya were used for the 3D modeling of objects in the scene and applying textures. Audacity was used for recording and editing the narration and sound effects. All of the programming was done using C\# in Visual Studio within the Unity game engine. The teacher avatar animations were created with the Cinema Mocap 2 plugin for Unity (Cinema Suite 2018) using the Microsoft Kinect V2 (Microsoft 2018).

Initially, a scale model of the PART Lab was modeled in SolidWorks. Next, the model was imported into Maya to add photorealistic textures to all of the objects. The model then was optimized to increase rendering speed so that the frames per second were high enough for a comfortable VR experience. With Microsoft Kinect V2, the motions and

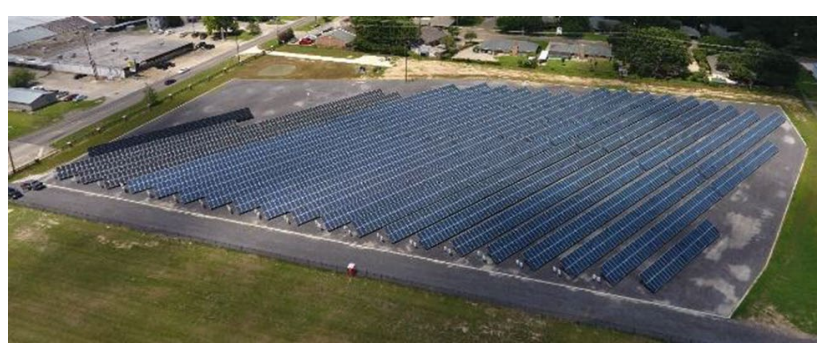

Fig. 2 PART Lab overhead view 
Fig. 3 Virtual Interactive Solar Instructor Robot (VISIR)
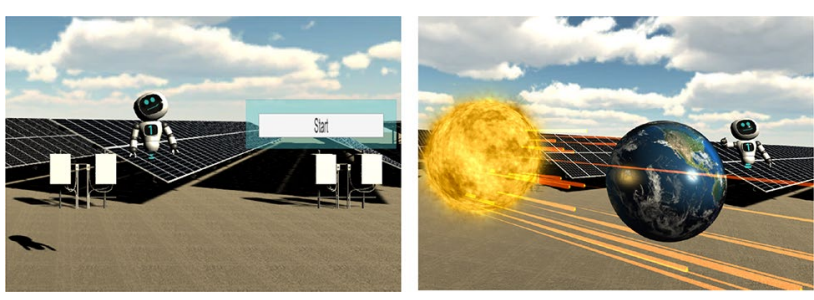

Fig. 4 Screenshots of PV-VR introduction (left) and solar resource on earth animation (right)

voice of the solar energy expert were captured and applied to the rigged virtual instructor avatar. The Virtual Interactive Solar Instructor Robot (VISIR), shown in Fig. 3, served as the guide throughout the application and was able to provide visual and vocal interaction with students during gameplay. The scenes of the application include a headset check, welcome introduction, and instruction on solar resource, PV power, inverters, three PV technologies (thin film, monocrystalline, and polycrystalline), measurement instruments, and the power and distribution network.

The design of an efficient VR training environment includes a combination of epistemology, pedagogy, methodology, and instructional strategy to impact the learner's perception, cognition, and actions (Babu et al. 2018). The application is designed to be used without external instruction, allowing the student to stay immersed until completed. At the start, there is a headset check where students are asked by VISIR if the visual and audio component are sufficient and if the headset is comfortable. The controls are explained with a diagram, although one-button controls are used to minimize difficulty. After confirming the headset is working properly, students are given a brief introduction of the PV-VR application and the actual PART Lab. The solar resource on Earth then is explained with accompanying animation, as shown in Fig. 4.

Following the solar resource animation, fundamentals on solar power generation were explained to students covering solar thermal power and PV. In the PV power area, an animation shows photons dislodging electrons to produce direct current (DC) (see Fig. 5a). Students then are directed to participate in a photon shooter activity in which photons are shot at the negative-doped silicon to free electrons that travel through a light bulb, turning it on (see Fig. 5b). Figure 5c

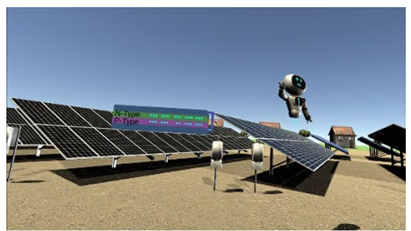

(a)

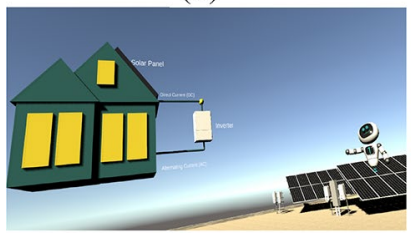

(c)

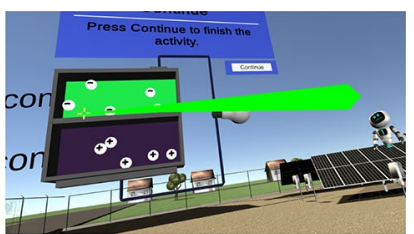

(b)

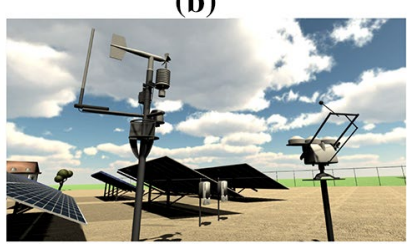

(d)
Fig. 5 Screenshots of the three-dimensional modeled environment include a direct current from photons animation, b photon shooter activity, $\mathbf{c}$ inverter animation, and $\mathbf{d}$ meteorological station and radiometer
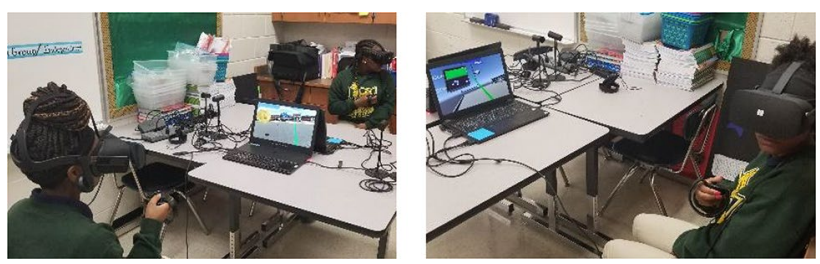

Fig. 6 Pilot study with Oculus Rift CR1 headsets

shows an animation where DC travels from the solar panels through the inverter creating AC to supply power to a home. Along with animation VISIR explains that most appliances run on AC, but solar cells generate DC. Therefore, inverters, running from solar cells, are necessary to convert DC to AC. The three different PV technologies at the PART Lab, thin film, monocrystalline, and polycrystalline, are explained with 3D models appearing in front of the participant. An assessment activity provides participants an opportunity to immediately apply what they learned. In the module activity (Fig. 9d), a close-up model was presented, while VISIR explains how each panel was produced along with the advantages and disadvantages of each one in terms of efficiency and cost.

\subsection{Pilot study}

A pilot test was conducted for qualitative feedback and suggestions to improve the application. The pilot test group consisted of forty-four 8th graders (18 males and 26 females). Students were given a headset and remote with no instructions and asked to complete the application. Two VR stations were used at the middle school shown in Fig. 6.

The application took between five and ten minutes to complete depending on how much the user looked around 
the environment without continuing to the next lesson or area. Laptops were used with the Oculus Rift CR1 headset for display and Oculus Touch remote for interaction. Following the application, students were given feedback surveys on the positive and negative aspects of the application and suggestions for improvement. Only two VR stations were used, so only two participants could perform the application at a time. This caused the testing time to be very lengthy, and as a result, not all students could be tested.

Due to this issue, the application was configured to be installed on the Oculus Go headset following the pilot study. The Oculus Go is a mobile all-in-one headset that does not need an external computer or power. The Oculus Go has a $2560 \times 1440$ fast-switch LCD (liquid crystal display) inside that can run at a refresh rate of $60 \mathrm{~Hz}$ or $72 \mathrm{~Hz}$. In comparison, the Oculus Rift CR1 uses one $1080 \times 1200$ OLED (organic light-emitting diode) display per eye for an effective resolution of $2160 \times 1200$ at a faster $90 \mathrm{~Hz}$. Therefore, the Oculus Go has a clearer image, but it will not feel as smooth as the Oculus Rift CR1. The Oculus Go uses insideout tracking, which is ideal for seated games and apps. The Oculus Rift CR1 has outside-in tracking and 6 degrees of freedom, which requires multiple external sensors that track the position of the headset and touch controls in a 3D space. This lets students not only adjust their view by rotating their heads, but they also can move around in space. Although the immersion quality is higher with the Oculus Rift CR1, it is more costly and less mobile, requiring an external computer and setup of multiple sensors.

For PV-VR to work in Oculus Go, the application needed to have a higher frame rate to run smoothly. All objects were optimized with three levels of detail (LOD) to reduce the total poly count. Some materials had many draw calls (a call to the graphics API to draw objects); therefore, they were changed to more simplistic materials. The trigger button on both devices is mapped the same; therefore, no controller modification was needed. Following the optimization, the PV-VR application was loaded onto the Oculus Go headsets and brought back to the school. Now, 10 students could perform the application at a time (see Fig. 7).

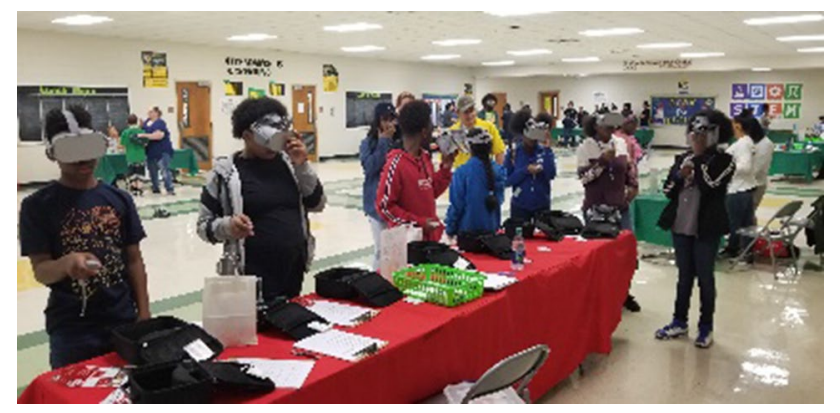

Fig. 7 Initial testing with Oculus Go headsets

\subsection{Developing $360^{\circ}$ panorama virtual environments}

Developing a $360^{\circ}$ panorama application is much more timeeffective than modeling objects as taking panoramas and inserting them into the scene is considerably quicker. To develop this application, $360^{\circ}$ panoramas are taken at the PART Lab using a YI 360 camera that was mounted on a tripod for the ground shots and mounted on a drone for the overhead shots. The $360^{\circ}$ panoramas are stitched together using YI 360 Studio and inserted into the Unity scene in the same locations that the camera as the user's point of view is located in the 3D modeled version, as shown in Fig. 8. The $360^{\circ}$ panoramic images are applied to the interior wall of a sphere, and the camera view is positioned in the center of the sphere to give the human eye the center viewpoint. Although the YI 360 camera shoots panoramas at $5.7 \mathrm{~K}$, the viewer can only see about a quarter of those pixels, or $1.4 \mathrm{~K}$, at any given moment. This means that in order to achieve $4 \mathrm{~K}$ in VR, one needs a $16-\mathrm{K} 360^{\circ}$ panorama (Redohl 2017). With current stitching software wasting half of the information, a multi-camera rig would need to capture more than $32 \mathrm{~K}$ to account for the loss of stitching overlap between cameras (Lee et al. 2016).

The same 3D models are used for the animations, closeup explanations, and activities, but the environment around the participant is a $360^{\circ}$ panorama instead of a $3 \mathrm{D}$ modeled VE. This allows for direct comparison of the two different VEs.

The interaction areas in both applications include the start area where instructions and safety precautions are given, the overview area (Fig. 9a) showing the user the entire field, and then five interaction areas shown in Fig. 8. The interaction areas include the solar resource area (Fig. 9b), an overview of PV power, three module types, inverters, the meteorological station and radiometer, and the power station that

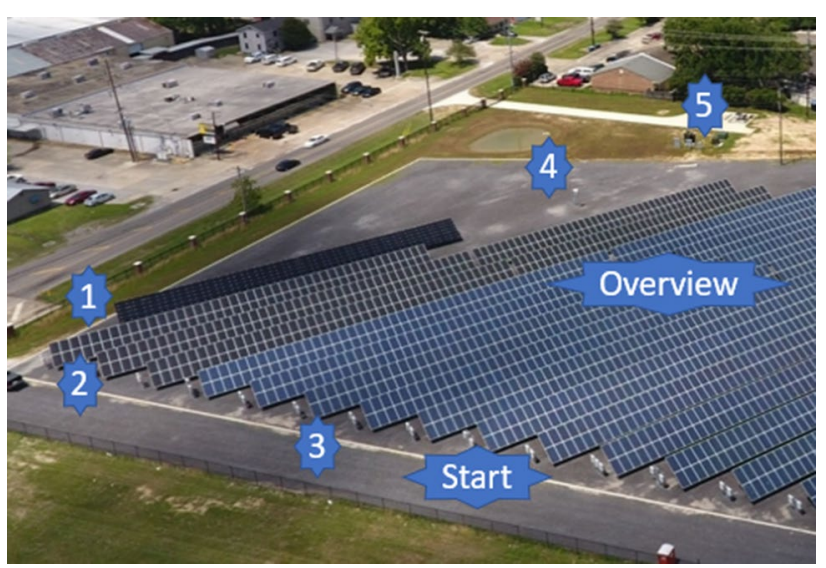

Fig. 8 Overhead view of participant interaction locations in the virtual environment 


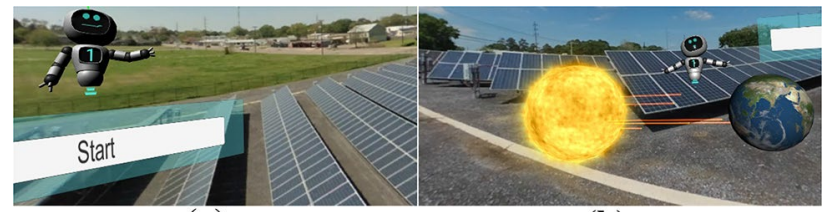

(a)

(b)

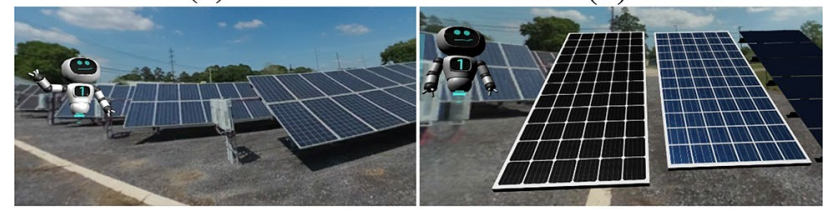

(c)

(d)

Fig. 9 Screenshots from the $360^{\circ}$ panorama application include a the photovoltaic-virtual reality overview, $\mathbf{b}$ an explanation of solar resource explanation, c polycrystalline PV modules, and d Module selection activity

includes two smart meters, a step-up transformer, a grounding transformer, and a Vista Switch. Along with getting a guided virtual tour, the user interacts with objects to aid in comprehension and to apply what VISIR is explaining. The photon shooter activity in Fig. 5b allows the user to shoot photons to dislodge electrons and allow current flow, which then powers a light bulb. The module selection activity shown in Fig. 9d tests participants to reinforce the learning content by having them choose the correct module type based on the characteristics given by VISIR.

\section{$2.4360^{\circ}$ panorama versus 3D modeled VE study}

For the comparative analysis, study participants were divided into two groups wherein they performed the following:

Steps for testing:

1. Take pretest

2. Start first VR application

(a) Group 1: $360^{\circ}$ panorama

(b) Group 2: 3D modeled VE

3. Take posttest

4. Take presence questionnaire

5. Start second VR application

(a) Group 1: 3D modeled VE

(b) Group 2: $360^{\circ}$ panorama

6. Take comparative analysis questionnaire

Group 1 performed the $360^{\circ}$ panorama version first and the 3D modeled VE second, while Group 2 went vice versa. Both groups completed each application, took preand posttests, a presence questionnaire, and a comparative
Table 1 Demographics of participants

\begin{tabular}{lll}
\hline Characteristics & Categories & Frequency (\%) \\
\hline Age & $18-19$ & 7 \\
& $20-21$ & 29 \\
& $22-23$ & 32 \\
& 24 and over & 32 \\
Gender & Male & 64 \\
& Female & 36 \\
Academic rank & Undergraduate & 93 \\
\multirow{3}{*}{ Educational background } & Graduate & 7 \\
& Engineering & 93 \\
& Science & 7
\end{tabular}

analysis questionnaire. The tests administered had six questions worth one point each for pre- and posttests. All questions were multiple-choice and were taken directly from the content presented in the application. Questions such as, "What happens when photons from the sun hit a solar module?" and "What type of module is made by deposition of layers of photovoltaic material on a substrate?" were used. The posttests were administered immediately after students finished the first application. Following the posttest, participants were given a presence questionnaire to measure the degree of immersion and realism offered by each application. The presence questionnaire, constructed by (Witmer and Singer 1998), consisted of four questions each on a 1 to 7 scale, with 1 being "not very often" and 7 being "very much so." Each question corresponds to a factor correlated with presence, such as "During the time of the experience, did you often think to yourself that you were actually in the application? During the experience I often thought that I was really standing in the application." The final presence score is the sum of the questions. Following the presence questionnaire, participants completed the other application and then took the comparative analysis questionnaire. This questionnaire consisted of eight questions aimed to gauge the contrast between the application types. Questions such as "Which gives you stronger spatial understanding" (for relative sizes and distances of objects) and "Which visual experience was more comfortable" were used where participants selected $360^{\circ}$ panoramas, 3D modeled VEs, or no difference. Finally, three open-ended questions were asked: one for feedback of positive aspects of the applications, one for negative aspects, and the last for any other comments or suggestions.

The sample size for this study was 28 university students. The resulting demographics are shown in Table 1. The participants were mostly undergraduate students, and all but two were enrolled in a solar design class. The enrolled subset had one semester of class in which they toured the actual PART Lab facility. 


\section{Results}

\subsection{Pilot testing results}

The application consisted of audible instruction, informative animations, and required game-like interaction to reinforce learning. The instructions were given in a way to minimize assistance needed with completing the application, and only three students out of the 44 tested needed assistance. Once students completed the application, they were given feedback surveys requesting their opinions of positive and negative aspects and suggestions for improvement. As shown in Table 2, 84\% of students gave positive comments on the VR experience and nearly $50 \%$ commented that it was fun.

Of the 44 students completing the application, $29 \%$ more females than males made positive comments about liking the VR experience. Thirty-five percent of females commented something positive regarding the immersiveness of the application, whereas no males commented on this. Fifteen percent more females commented on the application being informative and $14 \%$ more gave positive remarks on the gameplay or interaction. Students also were asked to give negative feedback, and the results are presented in Table 3.

The most common negative feedback was that the application was blurry, mentioned by 8 participants. Following this, five participants commented that the audio was too low, distracting background noises were heard, or there was an echo. Three participants mentioned that the application was confusing. Three males mentioned that there should be more surrounding objects, and three females mentioned that the headset was heavy. Only four participants had prior experience with VR systems, and two participants commented that they have VR systems at their house.

\section{2 $360^{\circ}$ panorama versus 3D modeled VE study results}

The average normalized gain has been used previously to measure the average effectiveness of an application in promoting conceptual understanding in a 6,000 student study

Table 2 Positive comments following application

\begin{tabular}{llll}
\hline Positive comments & \% of males & $\%$ of females & $\%$ Both \\
\hline Like virtual reality & 67 & 96 & 84 \\
Fun & 44 & 50 & 48 \\
Informative & 28 & 42 & 36 \\
Immersiveness & 0 & 35 & 20 \\
Gameplay & 17 & 31 & 25 \\
Like robot teacher & 11 & 19 & 16 \\
Graphics & 17 & 12 & 14 \\
\hline
\end{tabular}

Table 3 Negative comments following application

\begin{tabular}{llll}
\hline Negative comments & \% of males & \% of females & $\%$ Both \\
\hline Blurry & 17 & 19 & 18 \\
Audio/volume & 11 & 12 & 11 \\
Confusing & 6 & 8 & 7 \\
Add more surroundings & 17 & 0 & 7 \\
Too short & 0 & 12 & 7 \\
Headset heavy & 0 & 12 & 7 \\
Needs improvement & 6 & 4 & 5 \\
Scared to break & 6 & 4 & 5 \\
\hline
\end{tabular}

(Hake 1998). The average normalized gain $\langle g\rangle$ is defined as the ratio of the actual average gain (\% post- $\%$ pre) to the maximum possible average gain $(100 \%-\%$ pre $)$. The improvement on the posttest and the normalized gain for each application type is shown in Fig. 10.

The normalized gain for both applications is positive, demonstrating an improvement; however, the 3D modeled VE, 3 , is substantially lower than the $360^{\circ}$ panorama, 43 . The normalized gain of the $360^{\circ}$ panorama is in the medium gain region and the combined is in the low-gain region, according to Hake (1998). The Wilcoxon signed-rank test was performed across both applications and revealed that students showed improvement in the $360^{\circ}$ panorama condition ( $W=42.5, P<0.011)$, whereas the 3D modeled VR condition showed no significant improvement $(W=3.5$, $P=0.895$ ).

The results from the presence questionnaire are presented in Table 4. The questions are presented in the table, with the highest score being 7 for each question and a total of 28 for each application. The results clearly indicate that a high degree of realism was achieved by both applications, with the 3D modeled VE scoring slightly higher.

No significant difference between applications was found in each of the four presence questions using the Wilcoxon

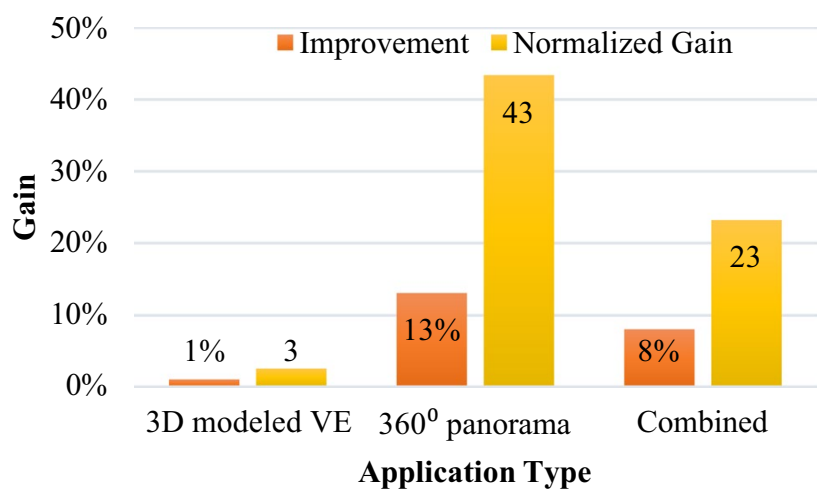

Fig. 10 Improvement and normalized gain for each application 
Table 4 Presence questionnaire results

\begin{tabular}{|c|c|c|c|}
\hline Question & Scale: $1-7$ & $\begin{array}{l}360^{\circ} \text { Panorama } \\
\text { mean (STD) }\end{array}$ & $\begin{array}{l}\text { 3D Modeled } \\
\text { VE mean } \\
\text { (STD) }\end{array}$ \\
\hline Please rate your sense of being in the application & Not at all/very much & $5.53(1.59)$ & $5.85(0.86)$ \\
\hline $\begin{array}{l}\text { To what extent were there times during the experience when the application } \\
\text { was the reality for you? }\end{array}$ & At no time/all the time & $4.73(1.73)$ & $5.15(1.10)$ \\
\hline $\begin{array}{l}\text { Which was strongest on the whole, your sense of being in the application, or } \\
\text { of being elsewhere? }\end{array}$ & $\begin{array}{l}\text { Being elsewhere/being in } \\
\text { application }\end{array}$ & $4.80(1.68)$ & $5.54(1.22)$ \\
\hline \multirow[t]{2}{*}{ Did you often think to yourself that you were actually in the application? } & Not often/very much & $5.53(1.45)$ & $5.85(1.03)$ \\
\hline & Total score & 20.60 & 22.38 \\
\hline
\end{tabular}

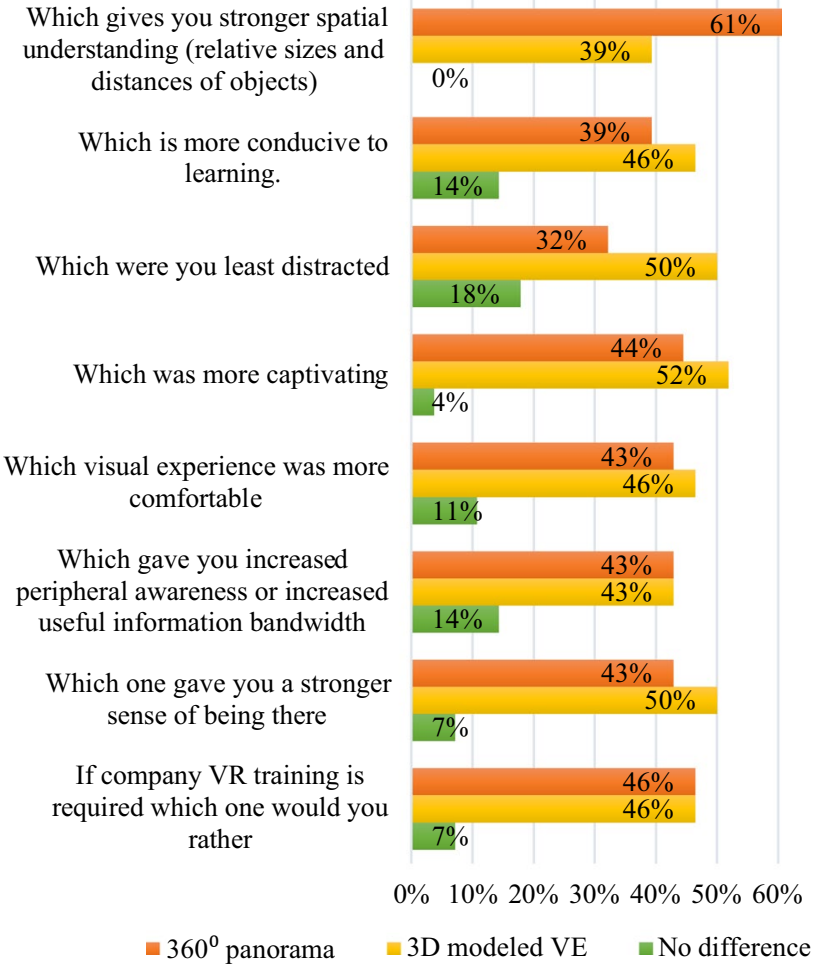

Fig. 11 Comparative analysis questionnaire results

signed-rank test for each of the matching pairs. Following the completion of both applications, participants were given a comparative analysis questionnaire, Fig. 11, to directly compare the applications. There were eight questions that are presented. As shown in Fig. 11, the largest contrast was the special understanding question with $360^{\circ}$ panorama at $61 \%$ and 3D modeled VE at 39\%. The 3D modeled VE scored higher on five of the other categories, with the highest being least distracted. Overall, the average on both applications was very similar with the 3D modeled VE having scored 3\% higher and an average of $9 \%$ claimed no difference.

The most common feedback on the open-ended questions for the two applications is shown in Table 5. The applications being informative were the most common positive comment amongst participants with $25 \%$ for the $360^{\circ}$ panorama and $14 \%$ for the 3D modeled VE application. The second most common positive comment with $25 \%$ for the $360^{\circ}$ panorama application being more realistic or photorealistic than the 3D modeled VE. No significant difference was found between applications in the comparative analysis results $(W=8.5, P=0.406)$.

The most common negative feedback involved low-quality pictures for the $360^{\circ}$ panorama application and lag when turning for the 3D modeled VE. The most common "other" comments also are listed, and there were several others common in both applications regarding the sound and the pauses VISIR makes in between explanations.

\section{Discussion}

In the pilot study, the overwhelming majority of students gave positive remarks regarding the VR experience, with nearly $50 \%$ stating that it was fun and over a third stating that it was informative. Students were excited and were primed for accepting VR training as a part of their educational

Table 5 Open-ended questionnaire results

\begin{tabular}{llll}
\hline Application type & Most common positive feedback & $\begin{array}{l}\text { Most common negative feed- } \\
\text { back }\end{array}$ & Most common “other" comments \\
\hline $360^{\circ}$ panorama & Informative, photorealistic & Low quality pictures & $\begin{array}{l}\text { Engaging, more potential } \\
\text { 3D modeled VE }\end{array}$ \\
\hline
\end{tabular}


curriculum. A previous study found males, rather than females, generally reported higher levels of the sense of actually being in the VE (Felnhofer et al. 2012). However, in this study the females indicated more of an appreciation of the VR experience than the males judging by comments made. Many users commented that if the headset was not lined up properly with their eyes, they experienced a blurry display. Indeed, if the headset is not sitting properly on the user's face, the display can appear blurry. In the application, the avatar asks users to adjust the headset; however, many of the students did not touch the headset. A few students commented that they were scared to break the headset and possibly feared adjustment might cause breaking. To address this issue, an animated headset adjustment scene was added to the beginning of the application.

In the comparative study, the 3D modeled VE group showed almost no improvement after completing the application with a normalized gain of 3 . In contrast, the $360^{\circ}$ panorama group showed a considerably higher normalized gain at 43 . Even though the 3D modeled group had slightly higher realism according to the presence questionnaire and had slightly higher averages in the comparative analysis, the effectiveness of the application was substantially lower according to the testing results. The improvement shown in the $360^{\circ}$ panorama group was found to be statistically significant using the Wilcoxon signed-rank test. No significant difference between applications was found in each of the four presence questions; however, larger sample sizes could show more contrast between applications. The lag when turning only was mentioned in the 3D modeled VE application and was the most common negative feedback. The 3D modeled VE application was more computationally expensive than the $360^{\circ}$ panorama version, because rendering all of the background objects required more processing time; this rendering would slow the frame rate enough for lag to occur (Reyna 2018). Although the objects were optimized and LODs were incorporated, the Qualcomm Snapdragon 821 processor in the Oculus Go headset is not powerful enough to produce the frame rate necessary for comfortable viewing in this application.

\section{Conclusion}

In this paper, a virtual training application, PV-VR, was developed and tested with quantitative and qualitative results presented. The initial pilot study obtained valuable feedback and suggestions that led to several improvements in the application with the headset check animation being the most notable. Switching to the Oculus Go saves money, increases mobility, decreases setup and breakdown time, and has less spatial requirements. This overall allows many more students to be tested at once, decreasing total testing time and increasing sample size potential. With the higher testing results and the higher majority of participants favoring the $360^{\circ}$ panorama application, it is the most effective for training and the quickest to develop. However, there are several limitations using $360^{\circ}$ panoramas as a medium, including image quality, static vantage point, and stitching parallax. The technology used to capture $360^{\circ}$ panoramas is still in its infancy, and the image quality is not comparable to traditional photography or videography. Another issue is that the panoramas have a static vantage point, which limits the exploration of the data to visual rotation and visual translation using videos. Also, for multiple camera rigs, the parallax between cameras may cause disturbing artifacts, such as misalignment and discontinuity. Even though the parallax issues can be managed to the point of being largely imperceptible, they currently are impossible to remove entirely. It is only a matter of time before consumer model $360^{\circ}$ cameras will be advanced enough to deliver the fidelity for a lifelike immersive experience in the classroom. New technologies should be continually tested to evaluate their possible impact on training and education.

\section{Future study}

The future goal of this study aims at evaluating the knowledge retention after immersive and non-immersive skill training methods. Actual field trip and virtual field trips also can be compared quantitatively and qualitatively. To increase student engagement, a system of rewards and goals to motivate students as well as feedback will be given with interactive cues that prompt learning.

Future updates with the PV-VR application will incorporate $\mathrm{PV}$ testing equipment at the PART Lab, including LED custom I-V flash test system, Atonometrics light soaking system, Therosensorik thermal imaging system, 32-channel daystar multitracer 5 , and custom angle of incidence tilt table. There also are plans to add various testbeds (see Fig. 12) for training and education as well as being able to experience them virtually before building.

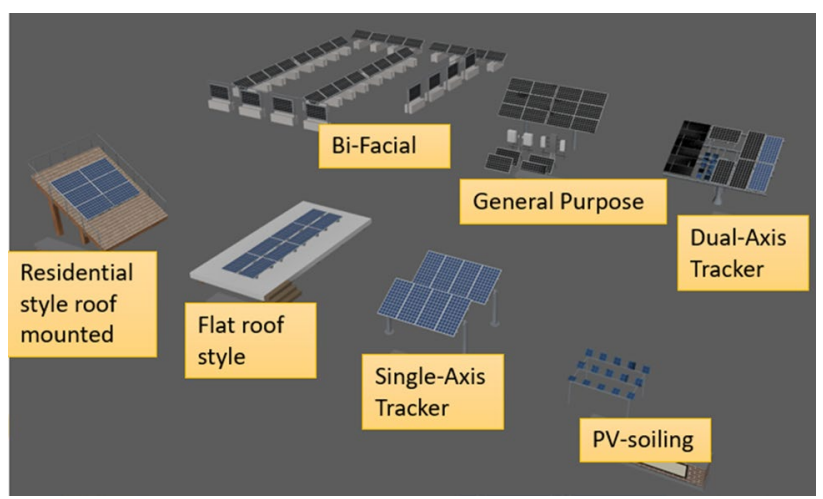

Fig. 12 Photovoltaic-virtual reality future testbeds 
Open Access This article is licensed under a Creative Commons Attribution 4.0 International License, which permits use, sharing, adaptation, distribution and reproduction in any medium or format, as long as you give appropriate credit to the original author(s) and the source, provide a link to the Creative Commons licence, and indicate if changes were made. The images or other third party material in this article are included in the article's Creative Commons licence, unless indicated otherwise in a credit line to the material. If material is not included in the article's Creative Commons licence and your intended use is not permitted by statutory regulation or exceeds the permitted use, you will need to obtain permission directly from the copyright holder. To view a copy of this licence, visit http://creativecommons.org/licenses/by/4.0/.

\section{References}

Abichandani P, McIntyre W, Fligor W, Lobo D (2019) Solar energy education through a cloud-based desktop virtual reality system. IEEE Access 7:147081-147093. https://doi.org/10.1109/ACCES S.2019.2945700

Alfalah SFM, Falah JFM, Alfalah T, Elfalah M, Muhaidat N, Falah O (2018) A comparative study between a virtual reality heart anatomy system and traditional medical teaching modalities. Virtual Reality. https://doi.org/10.1007/s10055-018-0359-y

Audacity (2019). https://www.audacityteam.org/. Accessed 13 Feb 2019

Autodesk (2019) Maya. https://www.autodesk.com/products/maya/ overview. Accessed 13 Feb 2019

Babu SK, Krishna S, Unnikrishnan R, Bhavani, R. (2018) Virtual reality learning environments for vocational education: a comparison study with conventional instructional media on knowledge retention. In: 2018 IEEE 18th international conference on advanced learning technologies (ICALT), pp. 385-389. https:// doi.org/10.1109/ICALT.2018.00094

Berg LP, Vance JM (2017) Industry use of virtual reality in product design and manufacturing: a survey. Virtual Reality. https://doi. org/10.1007/s10055-016-0293-9

Blender (2019). https://www.blender.org/. Accessed 13 Feb 2019

Borsci S, Lawson G, Broome S (2015) Empirical evidence, evaluation criteria and challenges for the effectiveness of virtual and mixed reality tools for training operators of car service maintenance. Comput Ind 67:17-26. https://doi.org/10.1016/j.compi nd.2014.12.002

Christopoulos A, Conrad M, Shukla M (2018) Increasing student engagement through virtual interactions: how? Virtual Reality 22(4):1-17. https://doi.org/10.1007/s10055-017-0330-3

Cinema Suite (2018) Cinema Mocap 2-markerless motion capture. https://assetstore.unity.com/packages/tools/animation/ cinema-mocap-2-markerless-motion-capture-56576. Accessed 26 April 2019

Cook M, Grime J (2020) Motivations, design, and preliminary testing for a $360^{\circ}$ vision simulator. Virtual Reality. https://doi. org/10.1007/s 10055-020-00433-x

Dassault Systèmes SolidWorks Corporation (2018) Solidworks, 3D CAD design software. https://www.solidworks.com/. Accessed 26 April 2019

Datallo A, Humer I, Tahai M, Sueda S, Pietroszek K, Eckhardt C (2018) Interactive large structure N-body gravity simulation for immersive learning in virtual reality. In: Immersive learning research network (ILRN), conference proceedings, pp 35-47. https://doi.org/10.3217/978-3-85125-609-3-09

Eiris R, Gheisari M, Esmaeili B (2018) Pars: using augmented 360-degree panoramas of reality for construction safety training. Int J Environ Res Public Health. https://doi.org/10.3390/ ijerph15112452
Eiris R, Gheisari M, Esmaeili B (2020) Desktop-based safety training using 360-degree panorama and static virtual reality techniques: a comparative experimental study. Autom Constr 109(102969):1-14. https://doi.org/10.1016/j.autcon.2019.10296 9

Felnhofer A, Kothgassner OD, Beutl L, Hlavacs H, Kryspin-Exner I (2012) Is virtual reality made for men only? Exploring gender differences in the sense of presence. In: Proceedings of the international society on presence research, Philadelphia, USA

Hake RR (1998) Interactive-engagement versus traditional methods: a six-thousand-student survey of mechanics test data for introductory physics courses. Am J Phys 66(1):64-74

Higuera-Trujillo JL, López-Tarruella Maldonado J, Llinares Millán C (2017) Psychological and physiological human responses to simulated and real environments: a comparison between photographs, $360^{\circ}$ panoramas, and virtual reality. Appl Ergon 65:398-409. https://doi.org/10.1016/j.apergo.2017.05.006

Jensen L, Konradsen F (2017) A review of the use of virtual reality head-mounted displays in education and training. Educ Inf Technol. https://doi.org/10.1007/s10639-017-9676-0

Klippel A, Zhao J, Jackson KL, La Femina P, Stubbs C, Wetzel R, Blair J, Wallgrün JO, Oprean D (2018) Transforming earth science education through immersive experiences-delivering on a long held promise. Br J Educ Technol 57:1-14

Kolb AY, Kolb DA (2005) Learning styles and learning spaces. Enhancing experiential learning in higer education. Acad Manag Learn Educ 4(2):193-212

Le QT, Pedro A, Park CS (2015) A social virtual reality based construction safety education system for experiential learning. J Intell Robot Syst Theory Appl 79(3-4):487-506. https://doi. org/10.1007/s10846-014-0112-z

Lee J, Kim B, Kim K, Kim Y, Noh J (2016) Rich360: Optimized spherical representation from structured panoramic camera arrays. ACM Trans Graph. https://doi.org/10.1145/28978 24.2925983

Li H, Chan G, Skitmore M (2012) Multiuser virtual safety training system for tower crane dismantlement. J Comput Civ Eng 26(5):638 647. https://doi.org/10.1061/(ASCE)CP.1943-5487.0000170

Loftus GR (1985) Observations: evaluating forgetting curves. J Exp Psychol Learn Mem Cogn 11(2):397-406. https://doi. org/10.1037/0278-7393.16.1.138

Merchant Z, Goetz ET, Cifuentes L, Keeney-Kennicutt W, Davis TJ (2014) Effectiveness of virtual reality-based instruction on students' learning outcomes in K-12 and higher education: a metaanalysis. Comput Educ 70:29-40. https://doi.org/10.1016/j.compe du.2013.07.033

Microsoft (2018) Microsoft kinect-windows app development. https ://developer.microsoft.com/en-us/windows/kinect. Accessed 26 April 2019

Moore HF, Eiris R, Gheisari M, Esmaeili B (2019) Hazard identification training using 360-degree panorama vs. virtual reality techniques: a pilot study. In: Computing in civil engineering 2019: visualization, information modeling, and simulation-selected papers from the ASCE international conference on computing in civil engineering 2019, pp 55-62. https://doi.org/10.1061/97807 84482421.008

Pereira RE, Gheisari M, Esmaeili B (2018) Using panoramic augmented reality to develop a virtual safety training environment. Proc Constr Res Cong 2018:29-39. https://doi.org/10.1061/97807 84481288.004

Pérez-Sabater CA, Montero-Fleta BE, Pérez-Sabater MA, Rising BE, De Valencia UP (2011) Active learning to improve long-term knowledge retention. In: Proceedings of the XII Simposio Internacional de Comunicación Social, pp 75-79

Pham HAIC, Dao N, Pedro A, Le QT, Hussain R, Cho S, Park CSIK (2018) Virtual field trip for mobile construction safety education 
using 360-degree panoramic virtual reality. Int J Eng Educ 34(4):1174-1191

Potkonjak V, Gardner M, Callaghan V, Mattila P, Guetl C, Petrović VM, Jovanović K (2016) Virtual laboratories for education in science, technology, and engineering: a review. Comput Educ 95:309-327. https://doi.org/10.1016/j.compedu.2016.02.002

Redohl S (2017) Explaining 360 video resolution: how to measure it, quality comparisons, and other metrics to look at. Immersive Shooter. http://www.immersiveshooter.com/2017/08/31/expla ining-360-video-resolution-how-measure-quality-comparisons/

Reyna J (2018) The potential of 360-degree videos for teaching, learning and research. In: INTED2018 proceedings, 1(March), pp 1448-1454. https://doi.org/10.21125/inted.2018.0247

Ritter KA III, Borst CW, Chambers TL (2018) Virtual solar energy center case studies. Comput Educ J 9(3):1-7

Rossetti T, Hurtubia R (2020) Are immersive videos close enough to reality? An assessment of the ecological validity of immersive videos in stated preference surveys. J Choice Model. https://doi. org/10.1016/j.jocm.2019.100198

Sacks R, Perlman A, Barak R (2013) Construction safety training using immersive virtual reality. Constr Manag Econ 31(9):1005-1017. https://doi.org/10.1080/01446193.2013.828844
Shu Y, Huang YZ, Chang SH, Chen MY (2019) Do virtual reality head-mounted displays make a difference? A comparison of presence and self-efficacy between head-mounted displays and desktop computer-facilitated virtual environments. Virtual Reality 23(4):437-446. https://doi.org/10.1007/s10055-018-0376-x

Unity 3D (2019) https://unity3d.com/. Accessed 13 Feb 2019

VRTK - Virtual Reality Toolkit (2018) Available at: https://vrtoolkit. readme.io/docs. Accessed 13 Feb 2019

Wang P, Wu P, Wang J, Chi H-L, Wang X (2018) A critical review of the use of virtual reality in construction engineering education and training. Int J Environ Res Public Health 15(6):1204. https://doi. org/10.3390/ijerph15061204

Witmer BG, Singer MJ (1998) Measuring presence in virtual environments: a presence questionnaire. Presence: teleoper. Virtual Environ. 7(3):225-240. https://doi.org/10.1162/105474698565686

Publisher's Note Springer Nature remains neutral with regard to jurisdictional claims in published maps and institutional affiliations. 